\title{
DERIVATION OF AN INPUT INTERVAL FIELD DECOMPOSITION BASED ON EXPERT KNOWLEDGE USING LOCALLY DEFINED BASIS FUNCTIONS
}

\author{
Maurice Imholz ${ }^{1}$, Dirk Vandepitte ${ }^{1}$, and David Moens ${ }^{1,2}$ \\ ${ }^{1}$ Department of Mechanical Engineering, Division PMA, Katholieke Universiteit Leuven \\ Celestijnenlaan 300B, 3001 Heverlee, Belgium \\ maurice.imholz@kuleuven.be - dirk.vandepitte@kuleuven.be \\ ${ }^{2}$ Department of Mechanical Engineering, Mechanical Engineering Technology TC, KU Leuven \\ Technology Campus De Nayer, Jan De Nayerlaan 5, 2860 Sint-Katelijne-Waver
}

Keywords: interval analysis, interval fields, uncertainty modelling

\begin{abstract}
In uncertainty calculation, the inability of interval parameters to take into account mutual dependence is a major shortcoming. When parameters with a geometric perspective are involved, the construction of a model using intervals at discrete locations not only increases the problem dimensionality unnecessarily, but it also assumes no dependency whatsoever, including unrealistic parameter combinations leading to possibly very conservative results. The concept of modelling uncertainty with a geometric aspect using interval fields eliminates this problem by defining basis functions and expressing the uncertain process as a weighted sum of these functions. The definition of the functions enables the model to take into account geometrically dependent parameters, whereas the coefficients in a non-interactive interval format represent the uncertainty. This paper introduces a new type of interval field specifically tailored for geometrically oriented uncertain parameters. The field has a non-interactive interval parameter in each node of the FE mesh to keep the true dimensionality of the uncertainty intact, but it obeys a bound on the gradient of the field to account for the dependency within the field.
\end{abstract}




\section{INTRODUCTION}

Non-deterministic FE-analysis has been subject of extensive research for quite some time. Certainly for reliability purposes, recognizing and quantifying sources of uncertainty is very important. The first type of non-deterministic FE-analysis that was introduced, stochastic FEanalysis, aimed at determining stochastic moments of some output quantities of the FE-analysis based on knowledge of the stochastic moments of the model input and/or parameters. The technique has been well established in literature (see e.g. $[1,2]$ ) and it has been applied to a variety of problems, see [3,4]. Thorough application of probabilistic theory may be cumbersome, mainly because of the increased computational cost and general lack of knowledge of stochastic parameters. Literature has shown that many authors content themselves with assumptions on the type of probability density functions or on its parameters before applying stochastic FE [5]. The Gaussian distribution is commonly used, but having infinite tails, the Gaussian distribution function assigns a non-zero probability to physically implausible values (e.g. negative densities). In addition to this, in reliability assessment the tails of the distribution are of greater interest as extreme conditions that make or break a design are usually situated there.

\subsection{Possibilistic Uncertainty Analysis in FE-modelling}

To cope with the drawbacks of stochastic FE, possibilistic FE-analysis was introduced [6, 7]. Instead of using ill-known stochastic parameters, uncertainty on model input or model parameters is modelled using possibilistic parameters, of which intervals, fuzzy numbers and convex regions are the most common. An interval models uncertainty on a variable as two extreme bounds which are not to be exceeded. A fuzzy number [8] is an extension to this concept, by considering the membership of a parameter to a certain interval as a continuous function, given by a membership function $\mu$ that ranges from 0 to 1 . A value of 1 indicates the value is certainly part of the interval, whereas a value of 0 indicates that it is definitely not. Convex regions define an uncertainty region in a multidimensional space and they attempt to capture uncertainty on parameter combinations by convex shapes such as ellipses. [9] presents an extensive elaboration on the use of convex regions. Both intervals and fuzzy numbers have been introduced in FE analysis $[10,11]$ and they have been the subject of numerous publications [12-16] containing applications and improvements. This paper uses possibilistic modelling, and it is limited to simple interval parameters. Possibilistic uncertainty modelling is well applicable in early design stages where the degree of uncertainty is rather high, as less information is required to perform the analysis.

For the sake of completeness, the authors mention the existence of hybrid approaches to uncertainty quantification, combining stochastic and probabilistic principles. Examples are fuzzy random variables [17] and interval probabilities [18]. However, their application remains limited in comparison to the specialized approaches.

The paper exclusively discusses intervals to model uncertainty. Throughout this work, an interval parameter $x^{I}$ with upper bound $\bar{x}$ and lower bound $\underline{x}$ is represented in two ways:

- using the bounds themselves: $x^{I}=\langle\underline{x} \mid \bar{x}\rangle$ 
- using the centre point $x_{c}=\frac{\bar{x}+\underline{x}}{2}$ and the interval radius $r_{x}=\frac{\bar{x}-\underline{x}}{2}: x^{I}=\langle x\rangle_{x_{c}}^{r_{x}}$

Furthermore, vector quantities in general are printed in bold lower case characters, while matrices are noted using bold upper case characters. A vector containing interval parameters (i.e. an interval vector) is marked as $\boldsymbol{x}^{I}=\left[\begin{array}{llll}\left\langle x_{1}\right\rangle_{x_{c, 1}}^{r_{x, 1}} & \ldots & \left\langle x_{n}\right\rangle_{x_{c, n}}^{r_{x, n}}\end{array}\right]^{T}$.

\subsection{Interactive intervals}

FE models usually represent real physical entities, such as cars, construction components or buildings. The parameters of these models are allocated to elements which are associated to a certain position in the spatial domain. In most cases, material properties are taken to be constant over the entire model if a single material is used. The geometry of parts is usually captured in a few global quantities, such as the thickness of plate parts, the length and radius of cylindrical parts or the curvature of bent parts. Uncertainty quantification then pertains to identifying the uncertainty on the global material or geometric parameter. However, the production process may introduce material impurities that can cause the model parameters to vary in the spatial domain, leading to different values for different elements. This may affect the uncertainty of the output quantities, but it is not considered if the uncertain parameters are defined globally. To account for this, instead of a single globally defined parameter for the entire model, interval parameters are assigned to each element of the FE model, representing parameter variability in space.

This introduces a problem with respect to numerical analysis. In standard interval analysis methods (of which a survey can be found in [7]), the interval parameters of interest are assumed independent: within their respective interval, they can all take values independently. This approach is in conflict with the element-wise interval parameters in FE models, as intervals of nearby points can hardly be independent, as this would lead to physically implausible parameter realisations. The parameters have to be assumed interdependent, which in interval context is referred to as interactive intervals.

\subsection{Non-deterministic fields}

In fact, the spatially oriented parameters of FE models can be considered as fields, in which each field realisation is a vector specifying the values in each element. Fields can also be defined in a non-deterministic way. The theory of random fields (RF) [19] defines fields in a probabilistic way, with each field realisation to be a function of well-specified random parameters. For a field variable $x(\boldsymbol{r})$, spatial correlation is modelled through definition of the correlation length $L_{\rho}$, which allows the covariance between the field variable at position $\boldsymbol{r}_{1}$ and $\boldsymbol{r}_{2}$ to be written as:

$$
\operatorname{COV}\left(x\left(\boldsymbol{r}_{1}\right), x\left(\boldsymbol{r}_{2}\right)\right)=f\left(\left\|\boldsymbol{r}_{1}-\boldsymbol{r}_{2}\right\|, L_{\rho}\right)=\exp \left(-\frac{\left\|\boldsymbol{r}_{1}-\boldsymbol{r}_{2}\right\|}{L_{\rho}}\right)
$$

with $\left\|\boldsymbol{r}_{1}-\boldsymbol{r}_{2}\right\|$ denoting the Euclidian distance between positions $\boldsymbol{r}_{1}$ and $\boldsymbol{r}_{2}$. Equation (1) represents an exponentially decaying covariance, but other functions are used as well. To analyse RF numerically, the field is decomposed to de-correlate the parameters using e.g. the 
Karhunen-Loève expansion (KLE), which after truncation expresses the field as a finite sum of weighted basis functions, of which the weights are random uncorrelated variables. [20] provides some background on the use of RF in FE context.

Next to RF, interval fields (IF) were introduced by Moens et al. [21] to model nondeterministic field variables in a possibilistic context. Much like the KLE, interval fields are defined as a sum of weighted basis functions, in which the weights are interval parameters. The general equation of an IF is given in equation $2 a$.

$$
x^{I}(\boldsymbol{r})=\sum_{i=0}^{n} \alpha_{i}^{I} \cdot \phi_{i}(\boldsymbol{r})
$$

The functions $\phi_{i}(\boldsymbol{r})$ are deterministic functions of the spatial co-ordinate $\boldsymbol{r}, \alpha_{i}^{I}$ are simple interval parameters.

The use of IF can be exploited fully if the definition of $\alpha_{i}^{I}$ and $\phi_{i}(\boldsymbol{r})$ is done in such a way that the interval parameters $\alpha_{\mathrm{i}}^{\mathrm{I}}$ are all non-interactive. In this case, the dependency in the field is captured exclusively by the basis functions $\phi_{i}(\boldsymbol{r})$, whereas the uncertainty is captured only by the interval parameters $\alpha_{i}^{I}$. This separation enables efficient uncertainty propagation throughout all steps of the analysis to the final output quantities. [22] considers an analysis with a single interval parameter to model the interval field. However, when more interval parameters are necessary to model an uncertain field with some degree of correlation between parameters, a decomposition has to be applied which ensures the interval parameters are truly noninteractive.

The objective of this paper is to find a general IF definition that enables an analyst to model knowledge which he/she possibly has on the uncertainty of the application as straightforwardly as possible, while at the same time ensures this strict division of uncertainty and dependency between the $\alpha_{i}^{I}$ and $\phi_{i}(\boldsymbol{r})$.

\section{INTERVAL FIELD SPECIFICATION}

The starting point of any uncertainty analysis is the knowledge of the analyst. Being an expert at a specific application or product, the analyst may have some knowledge on the uncertainty present. Firstly, this section introduces the global uncertainty parameters which is used further on to define an IF decomposition. A second section zooms in the on element level in an FE mesh and discusses the implications of this choice of uncertainty parameters on the local uncertainty in an arbitrary point.

\subsection{Definition of global uncertainty parameters}

Consider a field variable $x(\boldsymbol{r})$. The value in each point is expressed as the sum of the mean field value $\mu_{x}$ and the deviation from the mean value in that point:

$$
x(\boldsymbol{r})=\mu_{x}+x(\boldsymbol{r})-\mu_{x}=\mu_{x}+s_{x}(\boldsymbol{r})
$$


where $\mu_{x}=\frac{1}{\Omega} \int_{\Omega} x(\boldsymbol{r}) d \Omega$, and $\Omega$ a measure for the range of the field domain (i.e. the length, surface, or volume). When the field variable is uncertain, both the mean value and the deviation are subject to uncertainty. Equation (3a) is then left unchanged, with the superscript $I$ added to the variables to indicate they are now interval parameters:

$$
x^{I}(\boldsymbol{r})=\mu_{x}^{I}+s_{x}^{I}(\boldsymbol{r})
$$

In this description, $\mu_{x}^{I}$ is a simple interval parameter as defined before, but $x^{I}(\boldsymbol{r})$ and $s_{x}^{I}(\boldsymbol{r})$ are both interval fields, i.e. a set of interactive intervals in each point of the field domain. For the deviation it is assumed that its absolute value is bounded by a specified value $s_{x, M}$. This indicates the field variable itself never exceeds the interval bounds $\left\langle\underline{\mu_{x}}-s_{x, M} \mid \overline{\mu_{x}}+s_{x, M}\right\rangle$ in any point. To introduce the spatial dependency within the field, it is assumed that the difference between two adjacent points of the field is bounded as well, as most physical parameters normally exhibit some smooth evolution. This obliges the field values in adjacent points to vary within reasonable limits. To be able to express this property in a continuous field, the first spatial derivative of the field is taken into account. This maximum derivative constraint is converted to an interval with its centre at 0 . Furthermore, the uncertainty is assumed to be homogeneous with respect to the spatial dimension, leading to an interval on the first derivative, the magnitude of which is constant over the entire domain. The bounds are given by a single value $\left.\frac{\partial x}{\partial r}\right|_{M}$, leading to the interval $\left\langle-\left.\frac{\partial x}{\partial r}\right|_{M}\left|\frac{\partial x}{\partial \boldsymbol{r}}\right|_{M}\right\rangle$. In summary, the interval field $x^{I}(\boldsymbol{r})$ in this section has four major parameters defining the uncertainty:

- The lower bound on the mean field value $\underline{\mu_{x}}$

- The upper bound on the mean field value $\overline{\mu_{x}}$

- The maximum absolute value of the deviation from the mean value $s_{x, M}$

- the maximum absolute value of the first derivative of the field $\left.\frac{\partial x}{\partial r}\right|_{M}$

\subsection{Implications on local uncertainty in FE meshes}

The spatial variation modelled using an interval on the first spatial derivative defines the type of interactivity between the uncertain field values in nearby points. Using a probabilistic approach, geometric dependency or interactivity in general translates to a conditional probability formulated as $p\left(x_{\boldsymbol{r}_{\boldsymbol{i}}} \mid x_{\boldsymbol{r}_{\boldsymbol{i}}+\boldsymbol{\delta} \boldsymbol{r}}=\hat{x}\right)$, which signifies the probability distribution of the field variable $x$ at position $\boldsymbol{r}_{\boldsymbol{i}}$, given the value at a position close to $\boldsymbol{r}_{\boldsymbol{i}}$. In a possibilistic approach, which is preferred when the nature of the probability distribution is unknown, this can be replaced by a conditional interval $I\left(x_{\boldsymbol{r}_{\boldsymbol{i}}} \mid x_{\boldsymbol{r}_{\boldsymbol{i}}+\boldsymbol{\delta} \boldsymbol{r}}=\hat{x}\right)$, which holds the range of all possible values the field variable can take at the position $\boldsymbol{r}_{\boldsymbol{i}}$ under the same condition. This conditional interval is illustrated in figure 1. As the value of the field at $\boldsymbol{r}_{\boldsymbol{i}}+\boldsymbol{d} \boldsymbol{r}$ changes (horizontal axis), the interval of possible values at $\boldsymbol{r}_{\boldsymbol{i}}$ changes as well. 


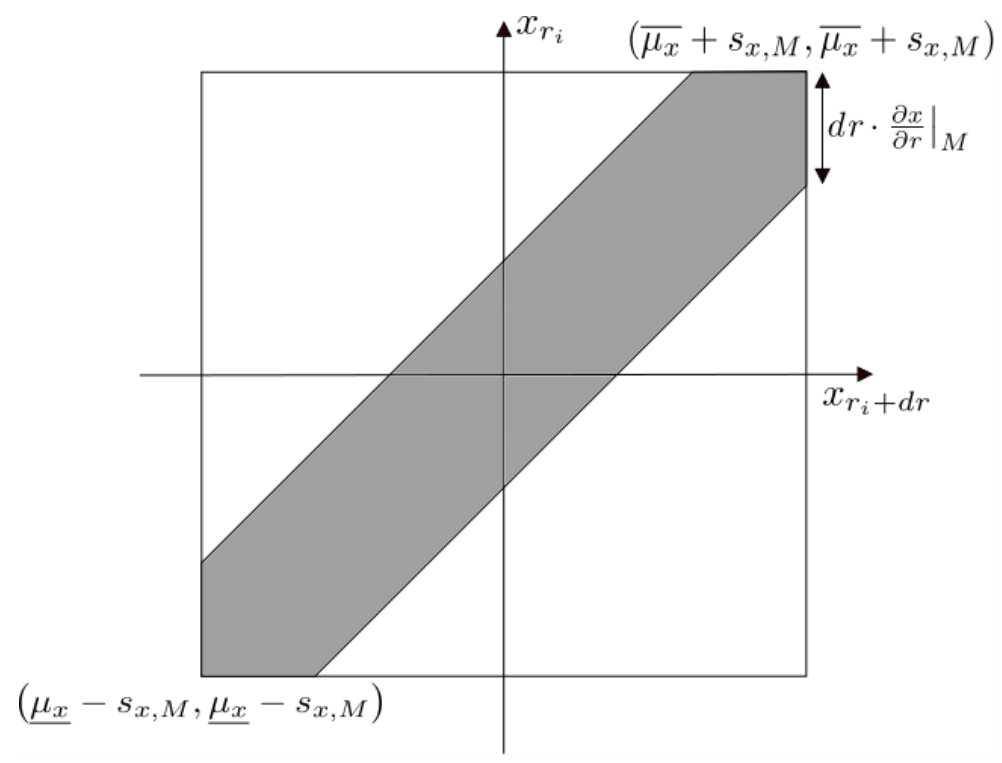

Figure 1: uncertainty region for two field points in close proximity: the grey area marks possible $\left(x_{\boldsymbol{r}_{i}}, x_{\boldsymbol{r}_{\boldsymbol{i}}+\boldsymbol{d r}}\right)$ combinations that satisfy the maximum gradient constraint. The square marks the uncertainty region if $x_{r_{i}}$ and

$$
x_{\boldsymbol{r}_{\boldsymbol{i}}+\boldsymbol{d r}} \text { are perfectly non-interatcive }
$$

In figure 1, $x_{\boldsymbol{r}_{\boldsymbol{i}}}$ and $x_{\boldsymbol{r}_{\boldsymbol{i}}+\boldsymbol{d r}}$ are scalar interval parameters at position $\boldsymbol{r}_{\boldsymbol{i}}$ and $\boldsymbol{r}_{\boldsymbol{i}}+\boldsymbol{d} \boldsymbol{r}$. The grey area represents possible combinations of $\left(x_{\boldsymbol{r}_{\boldsymbol{i}}}, x_{\boldsymbol{r}_{\boldsymbol{i}}+\boldsymbol{d r}}\right)$ according to the maximum gradient constraint. For non-interactive interval parameters, the domain of possible parameter combination would be the large square in figure 1. Clearly, the intervals are interactive as the uncertainty region is much smaller than the square. However, as the uncertainty region is a surface, it is still two-dimensional. If $n$ points of the field with values $x_{\boldsymbol{r}_{1}}, x_{\boldsymbol{r}_{2}}, \ldots, x_{\boldsymbol{r}_{\boldsymbol{n}}}$ are considered, the same principle applies: the domain of possible $\left(x_{\boldsymbol{r}_{1}}, x_{\boldsymbol{r}_{2}}, \ldots, x_{\boldsymbol{r}_{\boldsymbol{n}}}\right)$ combinations cannot be a perfect hypercube, but is still $n$-dimensional. this proves an important fact about the uncertainty modelling using the globally defined parameters mentioned above: the dimensionality of the uncertainty is equal to the number of discrete points in the field, or in FEcontext, the number of nodes.

\section{THE LOCAL INTERVAL FIELD DECOMPOSITION}

\subsection{Summary of objectives}

The starting point for this field decomposition is the explicit interval field description introduced by Moens et al. Equation (2a) expresses the field $x^{I}(\boldsymbol{r})$ as a weighted sum of basis functions, in which the weights are interval parameters:

$$
x^{I}(\boldsymbol{r})=\sum_{i=0}^{n} \alpha_{i}^{I} \cdot \phi_{i}(\boldsymbol{r})
$$

Next to this, we define the set of field realisations $X_{S}$ as:

$$
X_{S}=\left\{\tilde{x}(\boldsymbol{r}) \mid \tilde{x}(\boldsymbol{r})=\sum_{i=0}^{n} \tilde{\alpha}_{i} \cdot \phi_{i}(\boldsymbol{r}), \forall i: \tilde{\alpha}_{i} \in \alpha_{i}^{I}\right\}
$$


The analyst may specify the 4 previously defined global uncertainty parameters, which impose conditions on the field that have to be met. Each field realisation has to obey the following conditions:

1. $\underline{\mu_{x}} \leq \int_{\Omega} \tilde{x}(\boldsymbol{r}) d \boldsymbol{r}=\tilde{\mu}_{x} \leq \overline{\mu_{x}}$

2. $\forall \boldsymbol{r} \in \Omega: \tilde{x}(\boldsymbol{r})-\tilde{\mu}_{x} \leq s_{x, M}$

3. $\forall \boldsymbol{r} \in \Omega: \tilde{\mu}_{x}-\tilde{x}(\boldsymbol{r}) \geq s_{x, M}$

4. $\forall \boldsymbol{r} \in \Omega:-\left.\frac{\partial x}{\partial \boldsymbol{r}}\right|_{M} \leq \frac{\partial \tilde{x}(\boldsymbol{r})}{\partial \boldsymbol{r}} \leq\left.\frac{\partial x}{\partial \boldsymbol{r}}\right|_{M}$

In addition, the interval parameters $\alpha_{i}^{I}$ need to be non-interactive without violating this condition for any field realisation $\widetilde{x}(\boldsymbol{r})$.

To more easily comply with the first condition, the IF definition of equation (2a) is slightly altered to:

$$
x^{I}(\boldsymbol{r})=\mu_{x}^{I}+\sum_{i=0}^{n} \alpha_{i}^{I} \cdot \phi_{i}(\boldsymbol{r})
$$

If the interval $\mu_{x}^{I}$ is defined as $\mu_{x}^{I}=\left\langle\underline{\mu_{x}} \mid \overline{\mu_{x}}\right\rangle$, the first condition is automatically satisfied if

$$
\forall i: \int_{\Omega} \phi_{i}(\boldsymbol{r}) d \boldsymbol{r}=0
$$

This condition needs to be met by the basis functions defined in the next section.

The remainder of this section discusses the definition of the LIFD for a $1 D$ spatial domain, i.e. a variable which can vary along a line in space. However, all properties of the field can be extended to $2 \mathrm{D}$ and $3 \mathrm{D}$ domains, but the mathematical background then increases in complexity.

\subsection{Definition of basis functions}

In equation (2a), $\phi_{i}(\boldsymbol{r})$ are deterministic basis functions. For the purpose of propagating the interval field to obtain the output uncertainty, it is beneficial that the interval parameters $\alpha_{i}^{I}$ span a non-interactive interval space, since existing uncertainty propagation methods are based on non-interactive interval spaces. The basis functions possess the following properties to ensure this condition is met:

- All $\phi_{i}$ are piecewise second order polynomial functions so their first spatial derivative is continuous (see figure $2 \mathrm{~b}$ ).

- A single $\phi_{i}$ is positioned at each node at location $\boldsymbol{r}_{i}$ of the FE mesh, centered on this node.

- All $\phi_{i}$ are identically shaped

- All $\phi_{i}$ satisfy $\int_{\Omega} \phi_{i}(\boldsymbol{r}) d \boldsymbol{r}=0$

This leads to basis functions of the following type: 


$$
\phi_{i}\left(\boldsymbol{r}, \boldsymbol{r}_{i}\right)=f\left(\left\|\boldsymbol{r}-\boldsymbol{r}_{i}\right\|, a, R\right)
$$

Equation 6 indicates the $\phi_{i}$ are radial basis functions as their value only depends on the distance between the point $\boldsymbol{r}$ and the centre point of the function $\boldsymbol{r}_{i}$. In equation (6), $R$ is defined as the influence radius of the field, and $a$ the influence strength of the field. The influence radius determines the distance $\left\|\boldsymbol{r}-\boldsymbol{r}_{i}\right\|$ over which the function has a non-zero influence, whereas the influence strength is a scaling parameter that determines the impact of point $\boldsymbol{r}_{i}$ on its surroundings. Equation (7) gives the explicit definition of the basis functions for the onedimensional case. Figures $2 \mathrm{a}$ and $2 \mathrm{~b}$ are an illustration of equation (7) and give physical meaning to the parameters of equation (7). Figures $2 \mathrm{a}$ and $2 \mathrm{~b}$ show the shape of both the field variable itself and the first derivative.

$$
\phi_{i}(r)=C+a(1-C) \psi_{i}(r)=C+a(1-C)\left\{\begin{array}{cc}
0, & r<r_{i}-R \\
\frac{2\left(r-r_{i}+R\right)^{2}}{R^{2}}, & r_{i}-R \leq r \leq r_{i}-R / 2 \\
1-\frac{2\left(r-r_{i}\right)^{2}}{R^{2}}, & r_{i}-R / 2 \leq r \leq r_{i}+R / 2 \\
\frac{2\left(r-r_{i}-R\right)^{2}}{R^{2},} & r_{i}+R / 2 \leq r \leq r_{i}+R \\
0 \quad, \quad & r \geq r_{i}+R
\end{array}\right.
$$

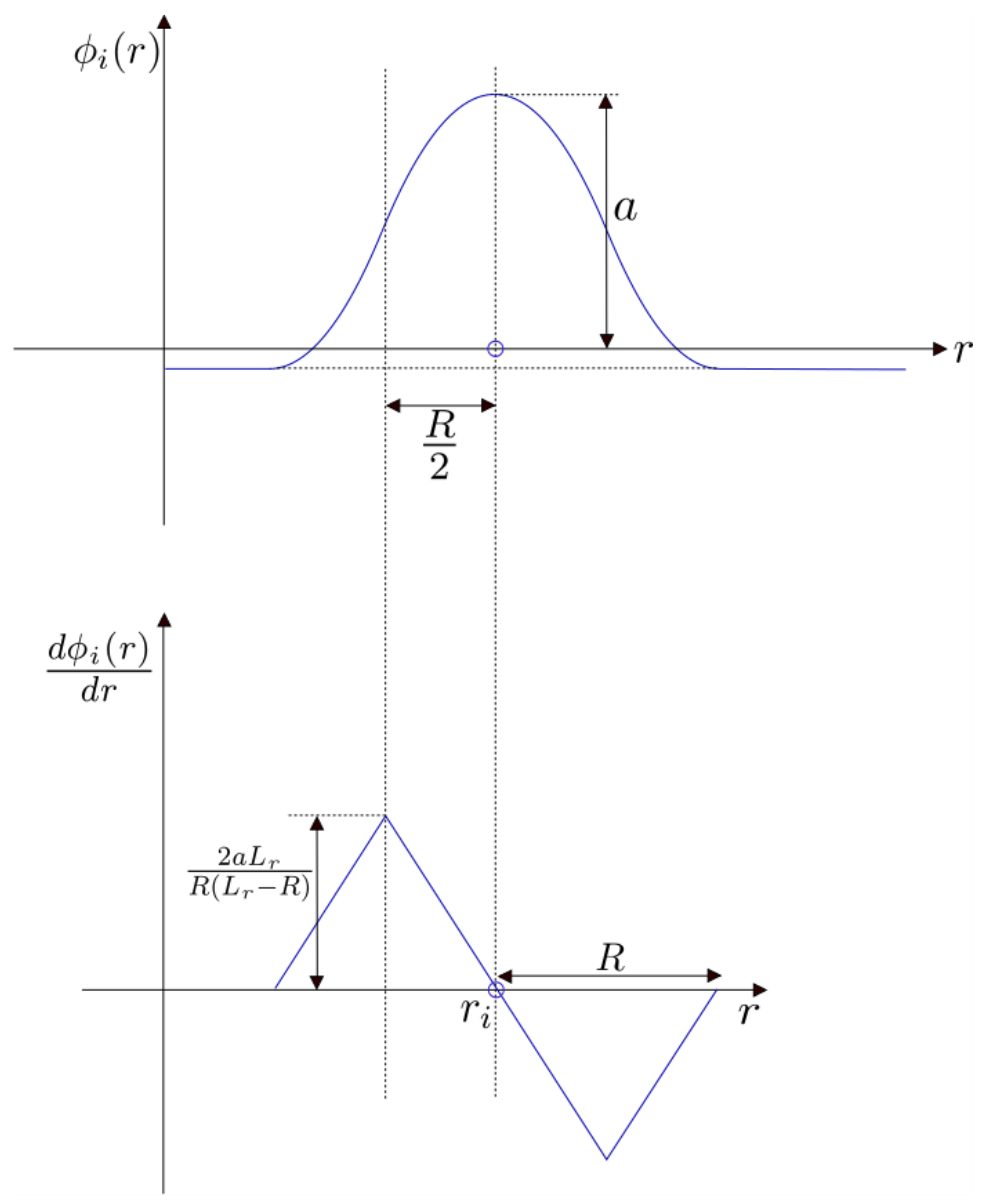

Figure $2 \mathrm{a}$ and $2 \mathrm{~b}$ : definition of a basis function and its first derivative 
$L_{r}$ is a measure for the range of the field, which in the 1D-case equals the length of the spatial domain (see figure 3). $C$ is a constant number which is used to make sure the basis functions satisfy $\int_{\Omega} \phi_{i}(\boldsymbol{r}) d \boldsymbol{r}=0 . C$ is derived as follows for the 1D-case:

$$
\begin{aligned}
\int_{0}^{L_{r}} \phi_{i}(\boldsymbol{r}) d \boldsymbol{r} & =\int_{0}^{L_{r}} C d r+a(1-C) \int_{0}^{L_{r}} \psi_{i}(r) d r \\
& =C \cdot L_{r}+a(1-C) \cdot R=0 \\
& \Leftrightarrow C=-a \frac{R}{L_{r}-R}
\end{aligned}
$$

The basis functions $\phi_{i}(r)$ are defined in each point along the field, as illustrated in figure 3 .

Figure 2a shows that the basis functions have a local character: beyond a radius $R$ their ability to change the field value vanishes, hence the name Local Interval Field Decomposition (LIFD).

Using this basis functions, the field is expressed as

$$
x^{I}(\boldsymbol{r})=\left\langle\mu_{x}\right\rangle_{C_{\mu}}^{R_{\mu}}+\sum_{i=1}^{N_{k}}\left\langle\beta_{i}\right\rangle_{0}^{1} \cdot \phi_{i}(\boldsymbol{r}, a, R)
$$

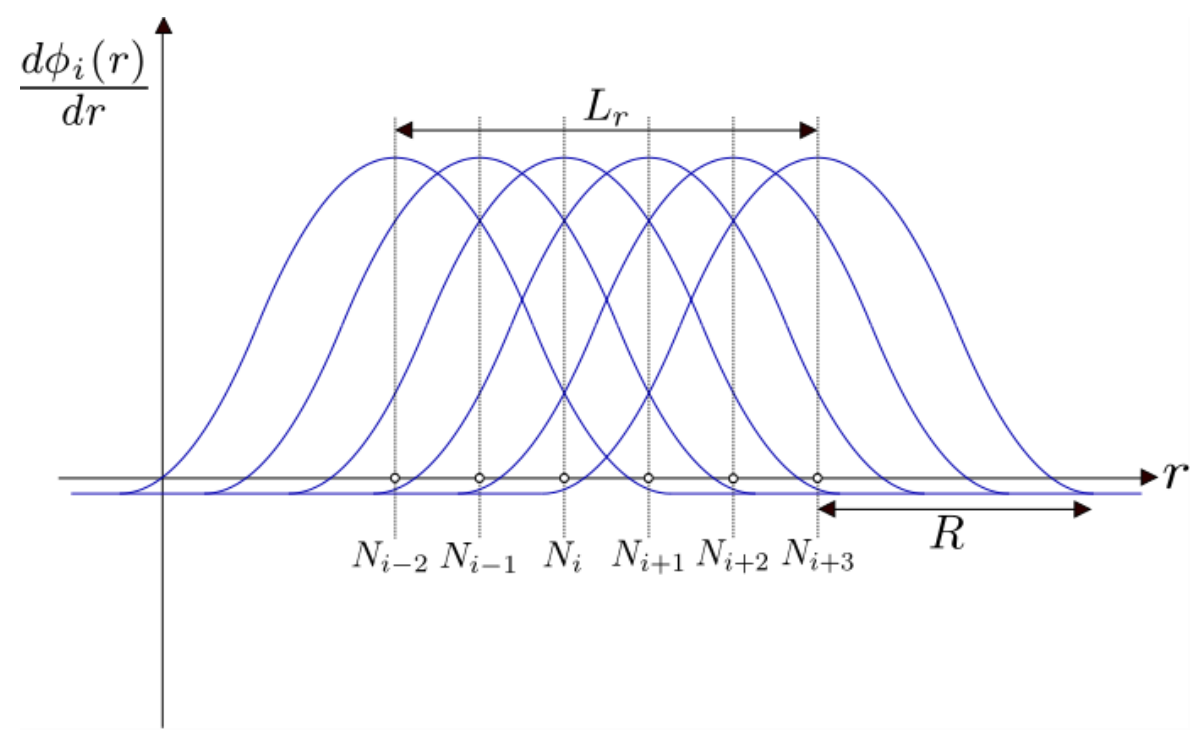

Figure 3: localisation of basis functions. Each point in the domain is allocated a separate similar basis function

In this definition, $\left\langle\mu_{x}\right\rangle_{C_{\mu}}^{R_{\mu}}$ is the interval on the average value of the field, with $R_{\mu}=\frac{\overline{\mu_{x}}-\underline{\underline{\mu_{x}}}}{2}$ and $C_{\mu}=\frac{\overline{\mu_{x}}+\underline{\mu_{x}}}{2} \cdot\left\langle\beta_{i}\right\rangle_{0}^{1}$ are defined in each node of a discretized field mesh with a total of $N_{k}$ discrete locations. They are all non-interactive normalised interval parameters. The four controllable parameters $a, R, R_{\mu}$ and $C_{\mu}$ of the interval field can be uniquely determined from the four global uncertainty parameters $\underline{\mu_{x}}, \overline{\mu_{x}}, s_{x, M}$ and $\left.\frac{\partial x}{\partial r}\right|_{M}$. For the derivation of equations 
(9a) to (9d) it is assumed that $R / 2 d r \in \mathbb{N}$ and $L_{r} / d r \in \mathbb{N}$, which is not necessary for the decomposition to work, but limits the derivation of the relations to this page.

$$
\begin{aligned}
\left.\frac{\partial x}{\partial \boldsymbol{r}}\right|_{M} & =2 \frac{2 a L_{r}}{R\left(L_{r}-R\right)}\left(1+2 \sum_{i=0}^{\lfloor R / 2 d r\rfloor} \frac{i \cdot 2 d r}{R}\right)=\frac{4 a L_{r}}{R\left(L_{r}-R\right)}\left(1+\frac{4 d r}{R} \cdot \frac{1}{2}\left(\frac{R}{2 d r}-1\right) \frac{R}{2 d r}\right) \\
& =\frac{2 a}{d r} \frac{L_{r}}{L_{r}-R} \\
s_{x, M} & =a+2 a\left(\sum_{i=0}^{[R / 2 d r\rfloor} \frac{2(i \cdot d r)^{2}}{R^{4}}+\sum_{i=0}^{\lfloor R / 2 d r\rfloor} 1-\frac{2(i \cdot d r)^{2}}{R^{4}}\right)+\sum_{i=1}^{R} a \frac{R}{L_{r}-R} \\
& =a+2 a\left(\sum_{i=0}^{R / 2 d r} \frac{2(i \cdot d r)^{2}}{R^{4}}-\frac{1}{2}+\sum_{i=1}^{R / 2 d r} 1-\frac{2(i \cdot d r)^{2}}{R^{4}}\right)+a \frac{L_{r}-2 R}{d r} \frac{R}{L_{r}-R} \\
& =a+2 a\left(\frac{R}{2 d r}-\frac{1}{2}\right)+a \frac{L_{r}-2 R}{d r} \frac{R}{L_{r}-R} \\
& =\frac{a R}{d r}+a \frac{L_{r}-2 R}{d r} \frac{R}{L_{r}-R} \\
\underline{\mu_{x}}= & C_{\mu}-R_{\mu} \\
\overline{\mu_{x}}= & C_{\mu}+R_{\mu}
\end{aligned}
$$

If the domain of the field is large compared to the influence radius of the basis functions, and the influence radius is large compared to the mesh discretisation step, simplified relations can be used, see equations (10a) to (10d):

$$
\begin{aligned}
& \left.\frac{\partial x}{\partial \boldsymbol{r}}\right|_{M}=\frac{2 a}{d r} \\
& s_{x, M}=\frac{2 a R}{d r} \\
& \underline{\mu_{x}}=C_{\mu}-R_{\mu} \\
& \overline{\mu_{x}}=C_{\mu}+R_{\mu}
\end{aligned}
$$

In these equations, $d r$ is the distance between points in which a basis function is defined. This quantity is set equal to the FE-mesh element size so that each node or element in the FEhas a basis function assigned to it. The reverse relationships in this case are given by equations (11a) to (11d):

$$
a=\left.\frac{\partial x}{\partial r}\right|_{M} \cdot \frac{d r}{2}
$$




$$
\begin{aligned}
& R=\frac{2 s_{x, M}}{\left.\frac{\partial x}{\partial \boldsymbol{r}}\right|_{M}} \\
& C_{\mu}=\frac{\overline{\mu_{x}}+\underline{\mu_{x}}}{2} \\
& R_{\mu}=\frac{\overline{\mu_{x}}-\underline{\mu_{x}}}{2}
\end{aligned}
$$

The field description of equation (2c) puts an interval $\left\langle\beta_{i}\right\rangle_{0}^{1}$ in each node/element of the FE mesh and therefore it does not reduce the dimensionality of the uncertainty. However, these intervals can now be considered as non-interactive while still obeying the maximum gradient condition. Making the step to a discretised field variable, suppose the vector $\boldsymbol{x}_{N_{k} \times 1}$ contains the field values in $N_{k}$ points of the spatial domain. In a context of uncertainty, this becomes an interval vector $\boldsymbol{x}_{N_{k} \times 1}^{I}$, which can be written as:

$$
\boldsymbol{x}^{I}=\boldsymbol{A} \cdot \boldsymbol{\beta}^{I}
$$

with $\boldsymbol{\beta}^{I}=\left[\begin{array}{lllll}\left\langle\mu_{x}\right\rangle_{C_{\mu}}^{R_{\mu}} & \left\langle\beta_{1}\right\rangle_{0}^{1} & \left\langle\beta_{2}\right\rangle_{0}^{1} & \ldots & \left\langle\beta_{N_{k}}\right\rangle_{0}^{1}\end{array}\right]$ the vector containing all interval parameters, and $\boldsymbol{A}$ the matrix with the discretised basis functions in its columns. Figure 4 illustrates how a global realisation of the field is obtained through adding all the basis functions according to equation (3b).

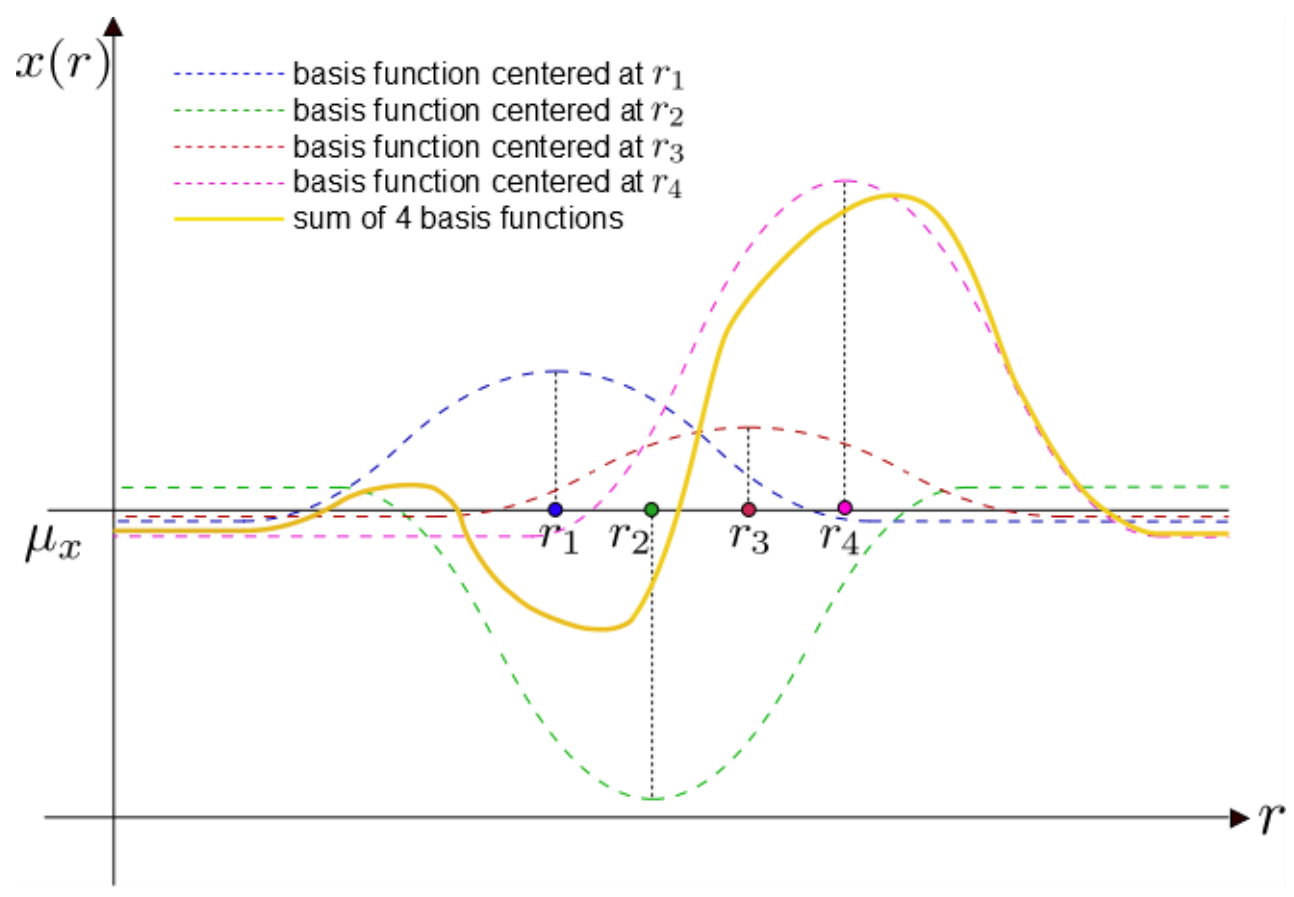

Figure 4: superposition of 4 basis functions to produce a single field realization

In figure 4, the dotted lines represent basis functions in four points with a random scaling factor within the interval $\langle-a \mid a\rangle$. Their sum is given by the solid yellow line. 


\section{3 basis functions adjustment at the edge of the domain}

The basis functions have a local character, but their definition is done globally: they all have the same shape, only their scaling factor can change, as figure 4 illustrates. However, this property cannot be held at the edges of the domain. If the globally defined maximum gradient condition has to be met at the edges, some adjustment has to be made. In practice, this problem affects all basis functions defined within a distance $R$ from the edge of the spatial domain.

The basis functions within distance $R$ of the edge are adjusted according to the process illustrated in figure 5. First, basis functions are added beyond the physical domain of the field that, according to their influence radius, still have a non-zero influence within the field. These are the non-blue lines in figure 5. The part of these functions that lie within the physical domain (right of the y-axis) are subtracted from the function at a distance $R$ to the right and the offset is adjusted to again make the average of each shape function equal to 0 . Figure 5 illustrates the adjusted basis functions for $R=4 d r$.

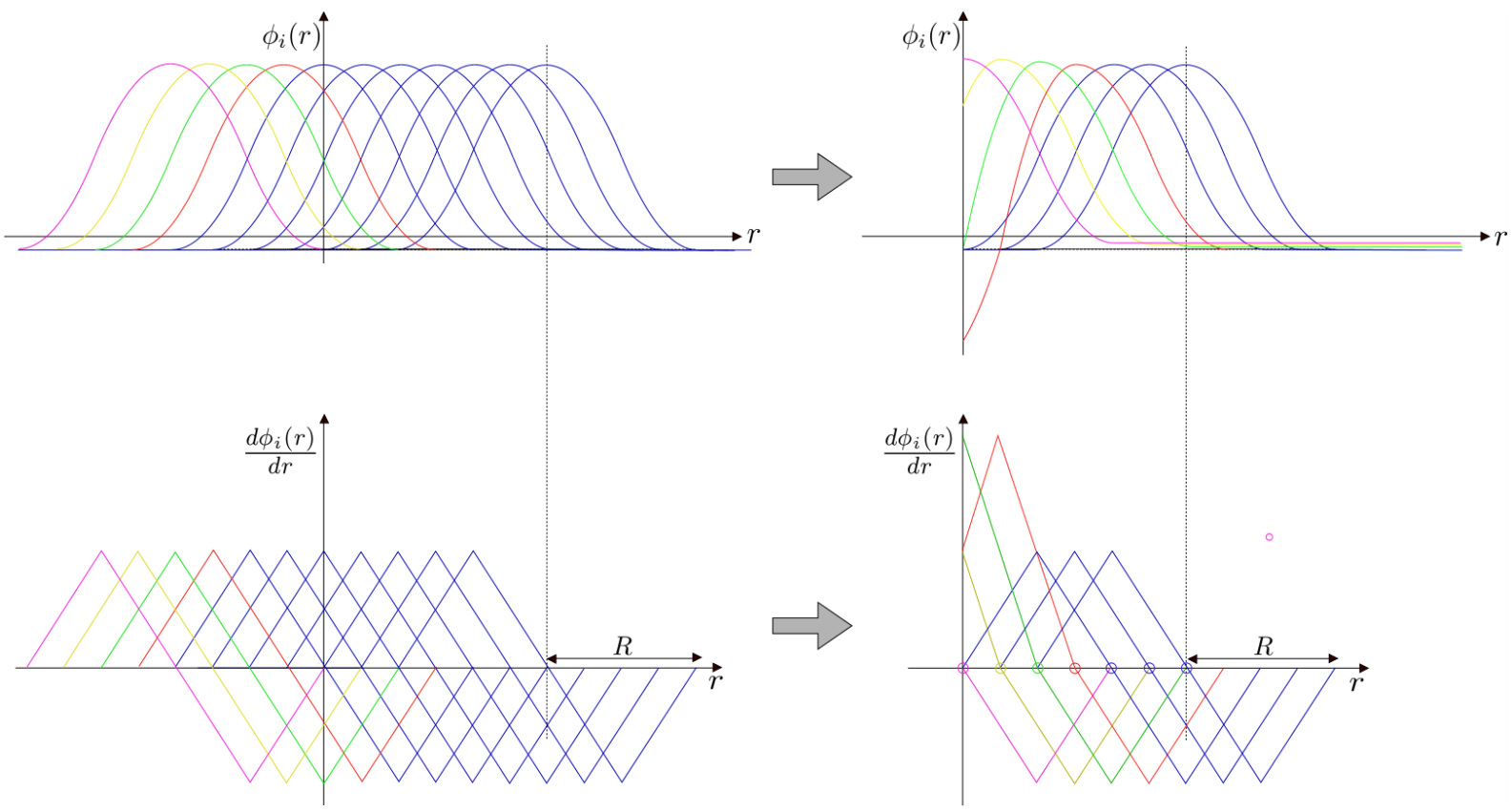

Figure 5: adjustment of the basis functions at the edge of the domain (the y-axis)

\section{4 basis function adjustment for deterministic measurement points}

Some quantities can be measured at certain positions, fixing the value at those points. From reliability perspective it is interesting to analyse the uncertainty on the output provided the measured values at these points are deterministic. Numerically, one could then determine possible points where measurements have to be taken to efficiently reduce the output uncertainty.

In this case, not the spatial variability around a certain average value is modelled, but the spatial variability given this (quasi-)deterministic measurement(s). The uncertain field then comprises the set of field configurations that interpolate the measured points, still obeying the 
maximum gradient condition. The basis functions are now allowed to have a nonzero average value, so the constant term in equation (7) is omitted from the definition.

The field definition of equation (2c) is rewritten in the following form:

$$
x^{I}(\boldsymbol{r})=q(\boldsymbol{r})+a \sum_{i=1}^{N_{k}}\left\langle\beta_{i}\right\rangle_{0}^{1} \cdot \phi_{i}(\boldsymbol{r}, R)
$$

In equation $(2 \mathrm{~d}), q(\boldsymbol{r})$ is a deterministic function that interpolates the measured points. If all realisations of $a \sum_{i=1}^{N_{k}}\left\langle\beta_{i}\right\rangle_{0}^{1} \cdot \phi_{i}(\boldsymbol{r}, R)$ have value zero at the measurement points, the field itself interpolates the measurement points as well due to the predefined function $q(\boldsymbol{r})$, which we assume to be piecewise second order polynomial between the measurement points to keep the first derivative continuous.

To add this ability to the LIFD, all basis functions within a range $R$ from a measured point $r_{m}$ have to be adjusted, as these functions have a nonzero influence to the measured point which has to be set to zero. However, introducing a measured point should not violate the globally defined maximum gradient condition, so simply forcing the interval parameters in points within a range $R$ to zero is not sufficient. Figure 6 illustrates the process to determine the new basis functions in close proximity to the measured point. The part between the basis point and the measured point is kept intact, but it is translated along the vertical axis so that the value in the measured point is zero (part A). To the left, the piecewise parabolic function is scaled so the derivative is continuous between part $\mathrm{A}$ and $\mathrm{B}$ and the function decays to zero beyond a range $R$ (part $\mathrm{B}$ ). To the right, a piecewise parabolic function ensures the derivative is continuous in the measurement point (part $\mathrm{C}$ ). To ensure that the maximum gradient is not exceeded, the entire function is scaled so that the derivative at the measured point is the same as the original basis function (blue dotted line). Figure 7 shows all basis functions for the case $R=4 \cdot d r$. 


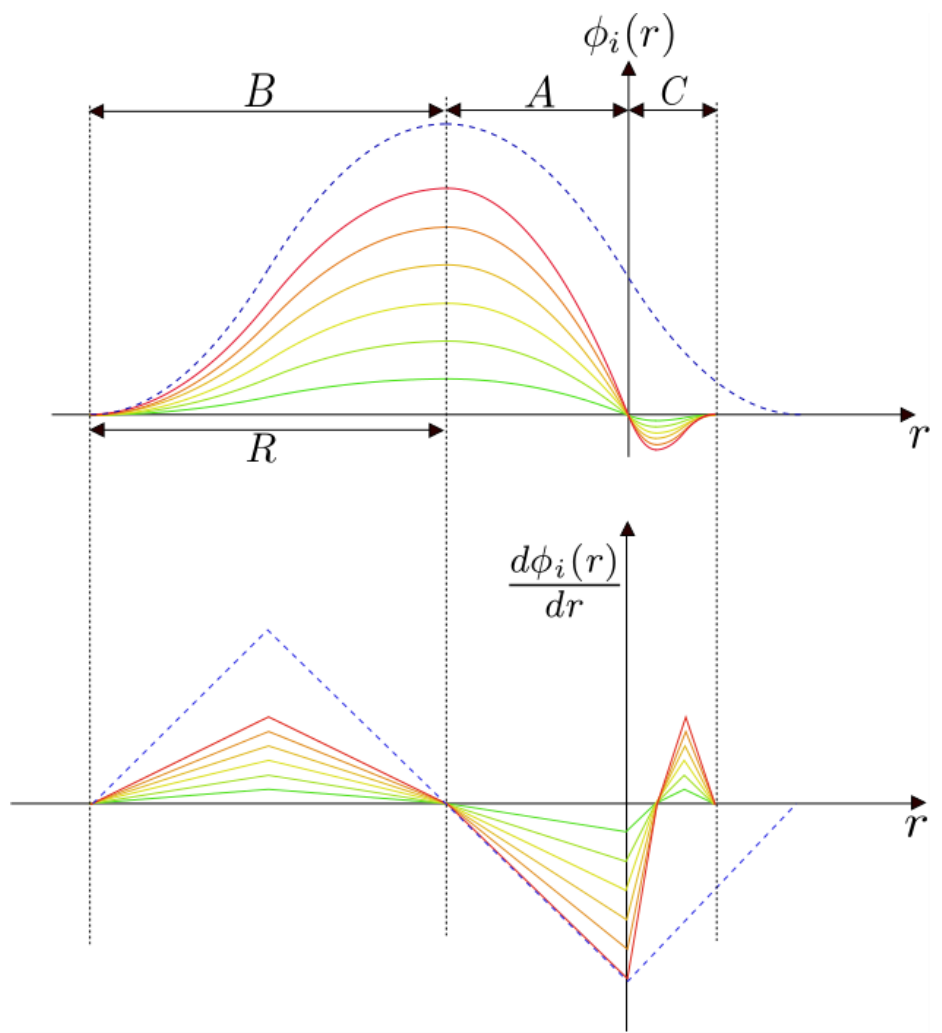

Figure 6: adjustment of the basis functions close to a measured point (the y-axis)

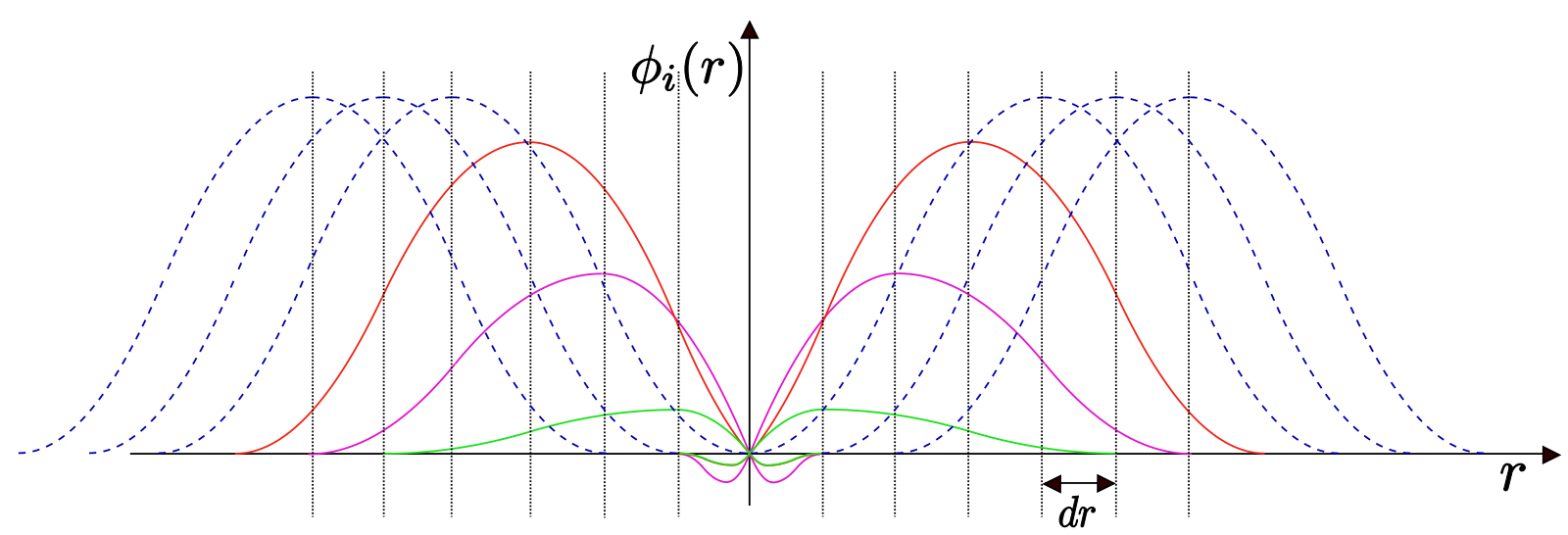

Figure 7: set of basis functions for $\mathrm{R}=4 \cdot \mathrm{dr}$. The measured point is located at the origin.

The purpose of interval analysis is to identify the output uncertainty by propagating a specific degree of input uncertainty. This is usually done by performing an optimization on the output function of interest over the uncertain input domain. The next section discusses this uncertain input domain after decomposition according to the LIFD and concerns some implications on the optimization process. 


\section{5 nature of the optimisation problem}

The main purpose of the LIFD is to define a non-interactive interval space in which the optimisation can be performed more easily to obtain uncertainty on the output quantities. The result of the LIFD is an $(n+1)$-dimensional non-interactive uncertainty space, comprised of:

- The interval on the average value $\left\langle\mu_{x}\right\rangle_{C_{\mu}}^{R_{\mu}}$ (determined by the global uncertainty parameters $\underline{\mu_{x}}$ and $\left.\overline{\mu_{x}}\right)$.

- The intervals $a \cdot\left\langle\beta_{i}\right\rangle_{0}^{1}=\left\langle\beta_{i}\right\rangle_{0}^{a}$ in each of the $n$ nodes of the FE-model (the sizes of which are determined by $a)$.

These $n+1$ interval parameters form a $(n+1)$-dimensional hypercubic uncertainty space. In this domain, the parameter combinations have to be found that produce extreme values on a specific output quantity. If this is done by a global optimization algorithm, as is common in interval analysis, this uncertainty space will also be the optimizing domain over which the extreme output values are determined. From this summary it is clear that the influence radius $R$ does not have an effect on the optimisation domain. The effect of $R$, and therefore the effect of $s_{x, M}$, is transferred to the optimisation function itself. Changing $R$ changes the nature of the dependency between the field values and lead to a new propagating function to the output.

Figure 8 shows the general framework of the LIFD. The first level transforms the four global uncertainty parameters to parameters of the field representation (equation (2c)). In the second level, three of these parameters determine the optimisation domain. Lastly, this domain is subjected to an optimization of a certain output function, which is partly determined by parameter $R$.

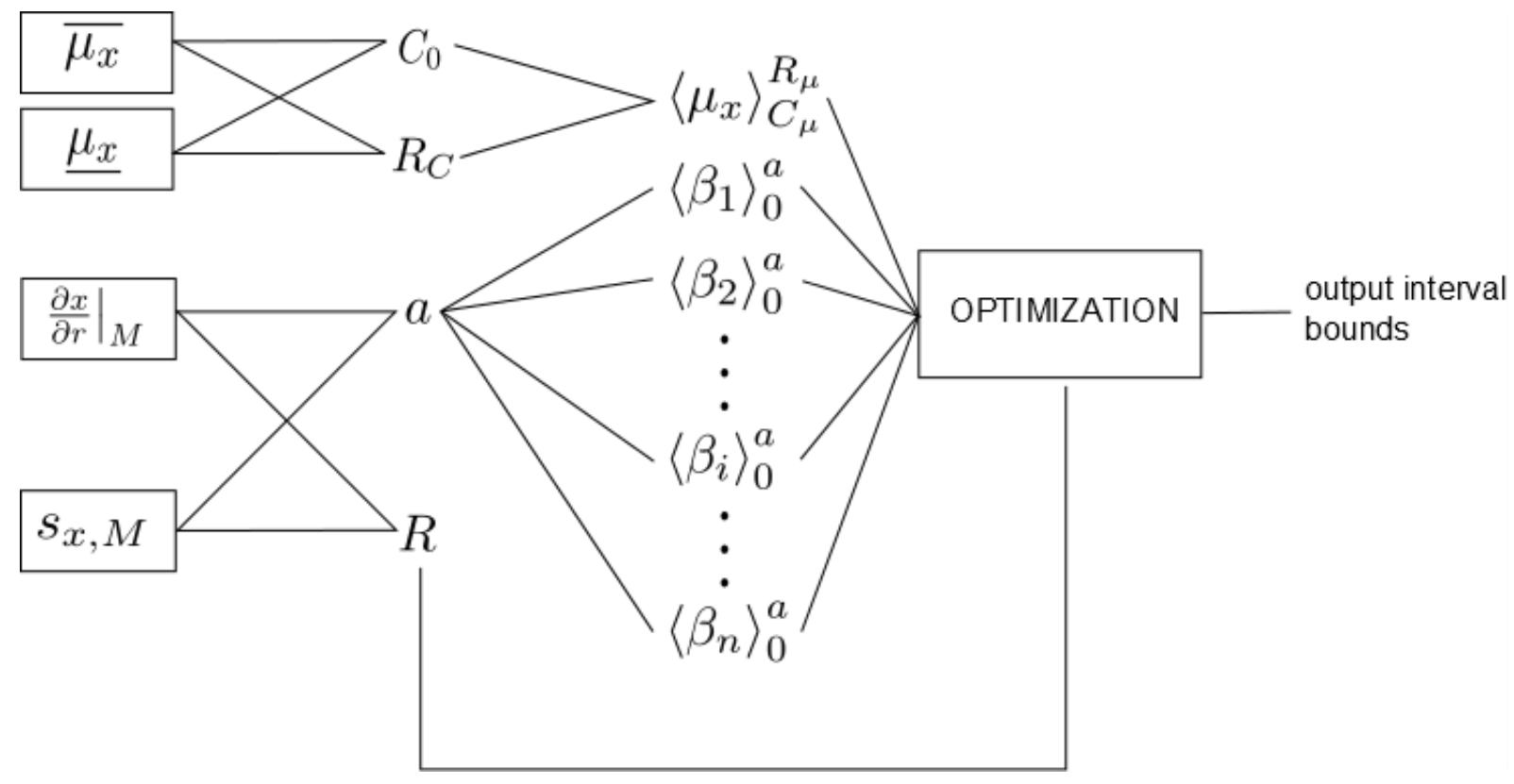

Figure 8: general blueprint of the field decomposition. 


\section{APPLICATIONS AND ILLUSTRATIONS}

In this section, the effect of $a$ and $R$ is illustrated in some numerical examples. A spatial domain $r$ is considered. For each choice of $(a, R, d r), 20$ samples are taken by sampling the interval parameters in a random vertex way (by taking each interval parameter either equal to $a$ or $-a$ ). The figures show the field variable itself, as well as the deviation from the mean value.
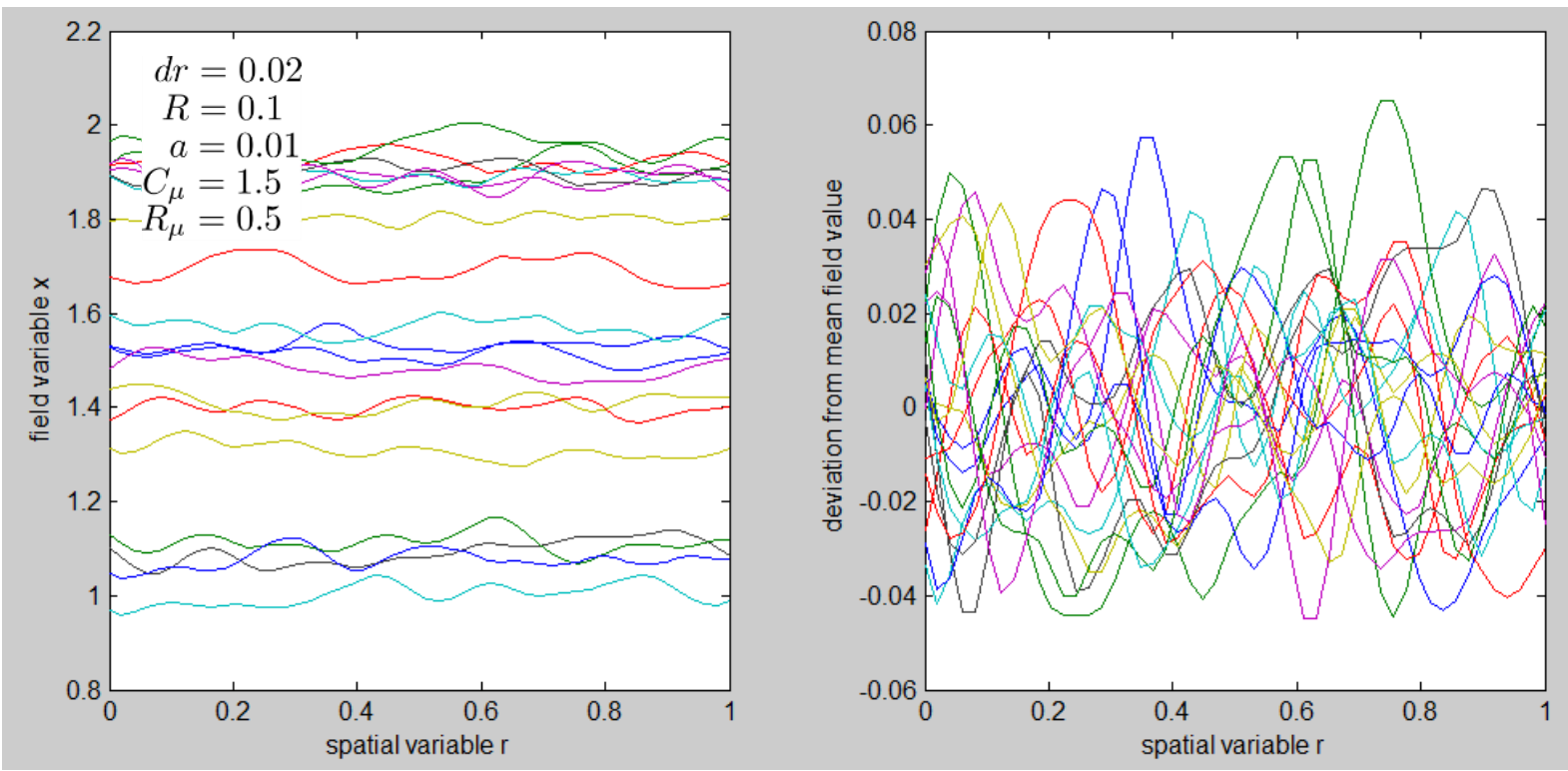

Figure 9a and 9b: field variable and deviation from the mean value
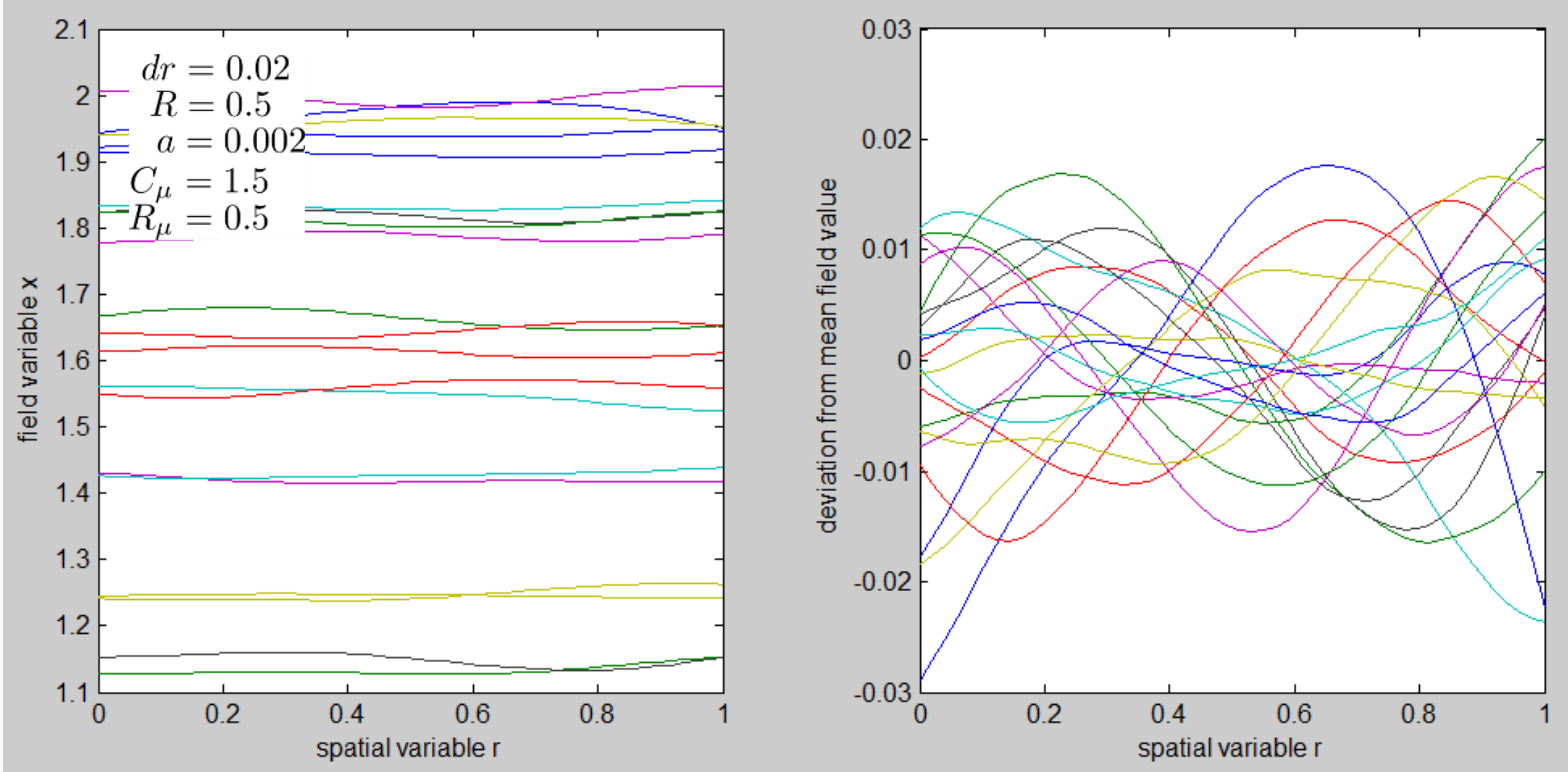

Figure 10a and 10b: field variable and deviation from the mean value

In figures 9 and 10 , the product $a R$ is kept constant, so the deviation from the mean is more or less the same. However, an increase of the value of $a$ increases the maximum gradient and produces more spiky realisations, as shown in figure $9 \mathrm{~b}$. Figure 11 has the same $R$-value as figure 10, which accounts for the general smoothness of the curves, however, the $a$-value is the same as in figure 9 , and so is the maximum derivative. The product of $a R$ is 5 times higher than 
in figures 10 and 11 , which can be seen from the deviation from the mean in figure $11 \mathrm{~b}$, which range is multiplied by 5 compared to figure $10 \mathrm{~b}$.
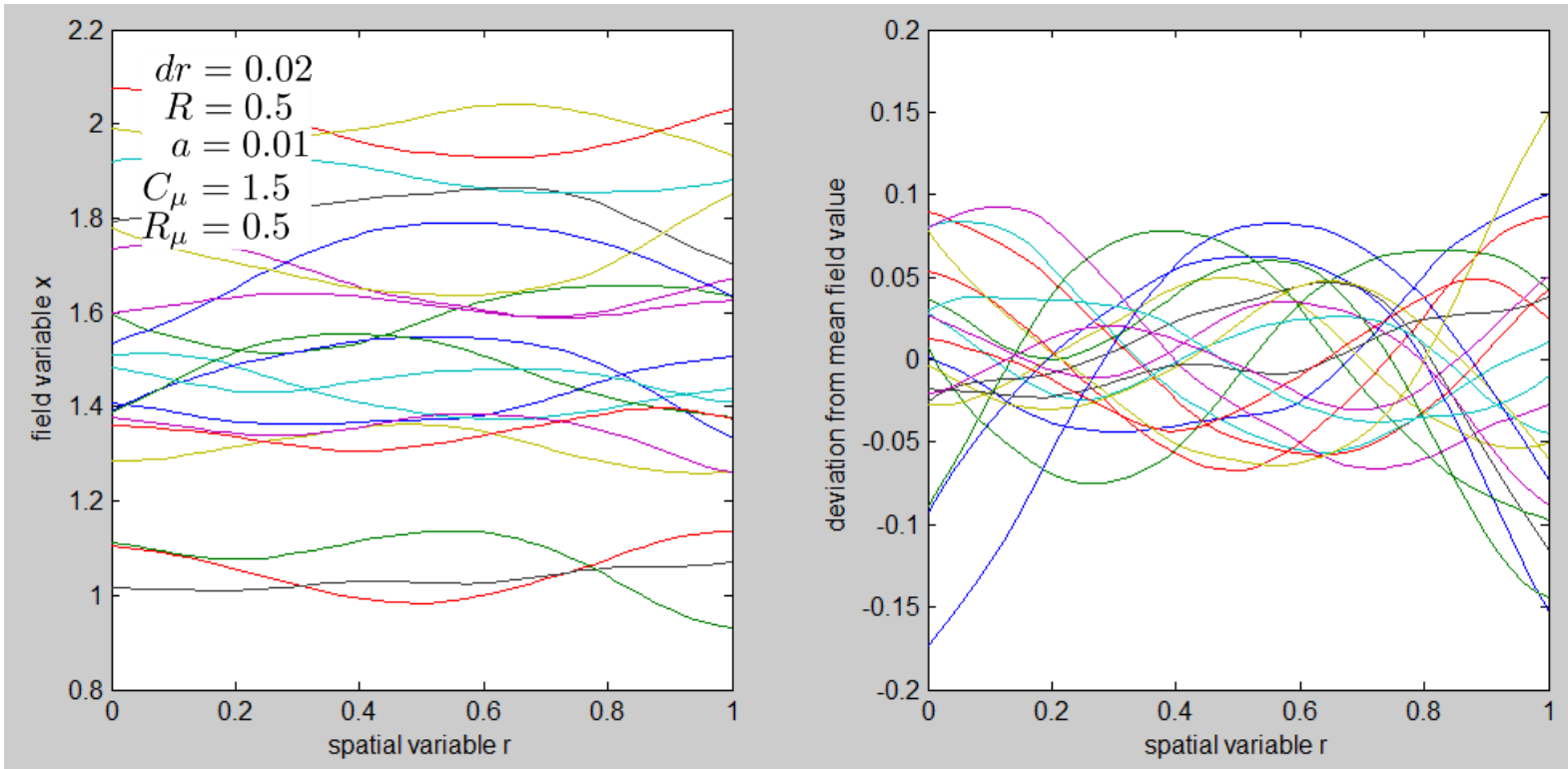

Figure 11a and 11b: field variable and deviation from the mean value
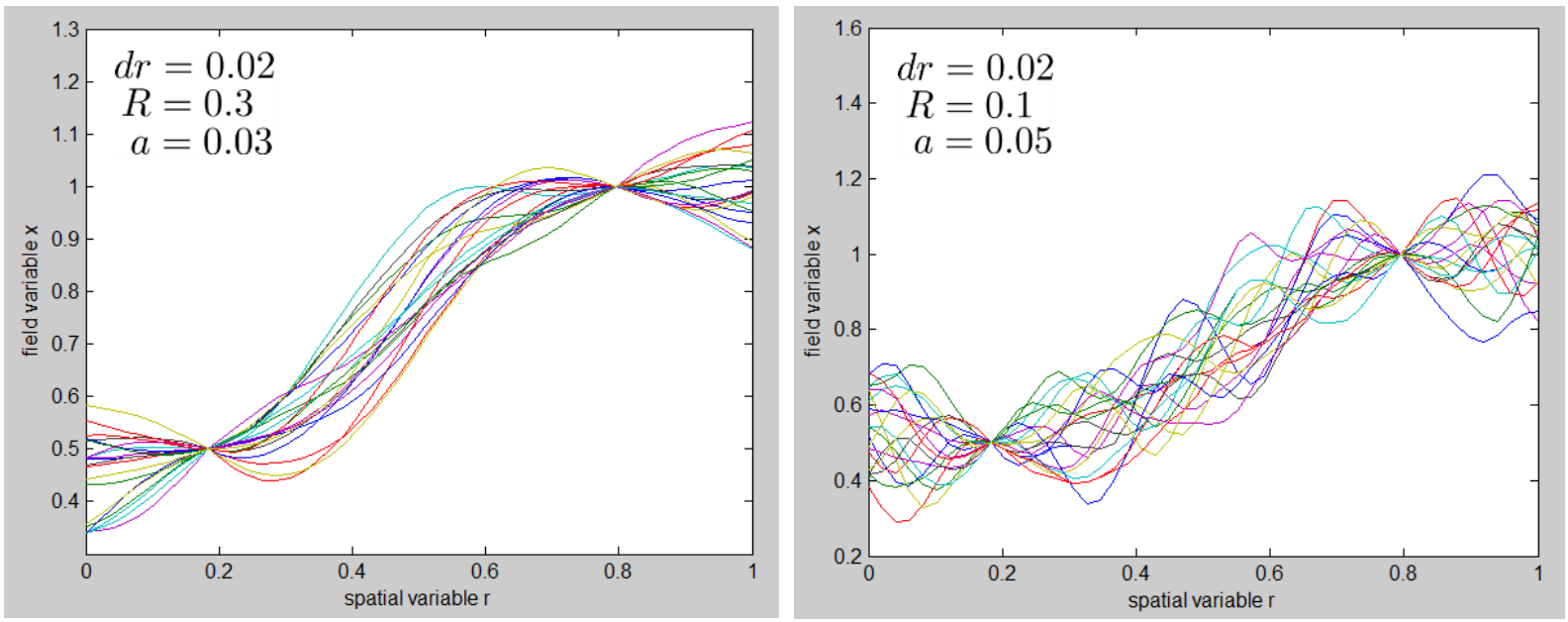

Figure $12 \mathrm{a}$ and $12 \mathrm{~b}$ : randomly generated field realizations using the measured-points-adjusted basis functions with a measured point at $r=0.2$ and $r=0.8$

Finally, figure 12 shows the field realisations when one measurement point is introduced halfway the spatial domain, for two sets of field parameters. All realisations now pass through the measured value, as required.

\section{CONCLUSIONS}

This paper introduces an interval field decomposition method that accounts for dependency between different points. The definition of the basis functions is based on the fact that, in order to achieve physically plausible field realisations, the value in each point is determined by the influence of the point itself and points in its immediate surroundings. This principle was 
converted to a bound on the gradient of the field, as well as a bound on the maximum deviation of the field from the average field value: these global uncertainty parameters can be translated to parameters of the local basis functions. This creates interval parameters that capture the uncertainty, but can be considered to be non-interactive while still obeying the dependency measure, i.e. the maximum gradient. This decomposition method enables the use of standard optimisation routines on a non-interactive parameter space, but at the same time, it allows for the application of sensitivity analysis because the transformed parameters retain their spatial location in the field. Note that the decomposition only concerns the dependency issue and does not reduce the dimensionality of the uncertainty. A second step in the analysis of uncertainty using this type of interval field is to create an efficient method to reduce the initial dimension down to a computationally plausible one, all with the purpose of efficiently determining the extreme field configurations within the predefined uncertainty. However, this process will be much easier as this field decomposition generates a non-interactive uncertain input space.

\section{ACKNOWLEDGEMENT}

The Research Fund KU Leuven and the Fund for Scientific Research - Flanders (F.W.O) are gracefully acknowledged for their support.

\section{REFERENCES}

[1] G. Stefanou, the stochastic finite element method: Past, present and future, Comput. Methods Appl. Mech. Engrg. 198 (2000) 1031-1051.

[2] A. Der Kiureghian, J-B. Ke, the stochastic finite element method in structural reliability, probabilistic Engineering Mechanics, 1988, Vol. 3 No. 2 83-91.

[3] A. Sluzalec, Stochastic Finite Elements in Optimization of Powder Metallurgy Materials, Mechanics Based Design of Structures and Machines: An international Journal, 40:1 (2012), 33-41.

[4] N. Scheerlinck, P. Verboven, J. Stigter, J. De Baerdemaeker, J. Van Impe; B. Nicolai, stochastic finite-element analysis of coupled heat and mass transfer problems with random field parameters, Numerical Heat Transfer, Part B: Fundamentals: An international Journal of Computation and methodology, 37:3 (200), 309-330.

[5] D. Vandepitte, D. Moens, Quantification of Uncertain and Variable Model Parameters in Non-Deterministic Analysis, IUTAM 2009, IUTAM Symposium on the Vibration Analysis of Structures with Uncertainties Vol. 27 (2011) 15-28

[6] I. Elishakoff, Y.J. Ren, Finite element methods for structures with large stochastic variations, Oxford Texts in Applied and Engineering Mathematics, Oxford, 2003.

[7] D. Moens, D. Vandepitte, A survey of non-probabilistic uncertainty treatment in finite element analysis, Comput. Methods Appl. Engrg. 194 (14-16) (2005) 1527-1555.

[8] L. Zadeh, Fuzzy sets, Informat. Control 8 (1965) 338-353. 
[9] Y. Ben-Haim, I. Elishakoff, Convex Models of Uncertainty in Applied Mechanics, Elsevier, New York, 1990.

[10] D. Moens, D. Vandepitte, A non-probabilistic finite element approach for structural dynamic analysis with uncertain parameters, $\mathrm{PhD}$ thesis, Katholieke universiteit Leuven, Departement werktuigkunde, Leuven, 2002.

[11] D. Moens, D. Vandepitte, Fuzzy Finite Element Method for Frequency Response Function Analysis of Uncertain Structures, AIAA Journal, Vol. 40 No. 1 (2002), 126136.

[12] W. Gao, interval finite element analysis using interval factor method, Comput. Mech (2007) 39: 709-717

[13] W. Desmet, D. Vandepitte, M. De Munck, W. Verhaeghe, D. Moens, recent advances in Interval and Fuzzy Finite Element Analysis, Proceedings of the $1^{\text {st }}$ international Symposium on Uncertainty Quantification and Stochastic Modelling, Maresias, São Sebastião, February-March 2012.

[14] M. De Munck, an adaptive Kriging based optimisation algorithm for interval and fuzzy FRF analysis, ISMA 2008, proceedings of the ISMA 2008 International Conference on Noise and Vibration Engineering, 3767-3776.

[15] W. Xiaojun, Q. Zhiping, Interval Finite Element Analysis of Wing Futter, Chinese Journal of Aeronautics 21 (2008) 134-140.

[16] S. Majundar, Sukanta Nayak, S. Chakraverty, Huzzy and Interval Finite Element Method for Heat Conduction Problem, International Journal of Advances in Applied Sciences, Vol. 1, No. 4 (2012), 171-180.

[17] M. Puri, D. Ralescu, Fuzzy Random Variables, Journal of Mathematical Analysis and Applications 1147 (1986) 409-422.

[18] K. Weichselberger, The theory of interval probability as a unifying concept for uncertainty, International Journal of Approximate Reasoning 24, 2-3 (2000) 149-170.

[19] E.Vanmarcke, Random Fields: analysis and synthesis, MIT Press, Cambridge, 1993.

[20] R. Ghanem, P. D. Spanos, Stochastic finite elements: a spectral approach, Springer, NewYork, 2003.

[21] D. Moens, M. De Munck, W. Desmet, D. Vandepitte, Numerical dynamic analysis of uncertain mechanical structures based on interval fields, IUTAM 2009, IUTAM Symposium on the Vibration Analysis of Structures with Uncertainties Vol. 27 (2011) 71-83.

[22] W. Verhaeghe, W. Desmet, D. Vandepitte, D. Moens, Interval Fields to represent uncertainty on the output side of a static FE analysis, Comput. Methods Appl. Mech. Engrg. 260 (2013) 50-62. 